\title{
Genome editing of human pancreatic beta cell models: problems, possibilities and outlook
}

\author{
Diego Balboa ${ }^{1,2} \cdot$ Rashmi B. Prasad ${ }^{3} \cdot$ Leif Groop $^{3,4} \cdot$ Timo Otonkoski $^{1,5}$ iD
}

Received: 18 January 2019 / Accepted: 25 April 2019 / Published online: 3 June 2019

(C) The Author(s) 2019

\begin{abstract}
Understanding the molecular mechanisms behind beta cell dysfunction is essential for the development of effective and specific approaches for diabetes care and prevention. Physiological human beta cell models are needed for this work. We review the possibilities and limitations of currently available human beta cell models and how they can be dramatically enhanced using genome-editing technologies. In addition to the gold standard, primary isolated islets, other models now include immortalised human beta cell lines and pluripotent stem cell-derived islet-like cells. The scarcity of human primary islet samples limits their use, but valuable gene expression and functional data from large collections of human islets have been made available to the scientific community. The possibilities for studying beta cell physiology using immortalised human beta cell lines and stem cellderived islets are rapidly evolving. However, the functional immaturity of these cells is still a significant limitation. CRISPRCas9 (Clustered Regularly Interspaced Short Palindromic Repeats/CRISPR-associated protein 9) has enabled precise engineering of specific genetic variants, targeted transcriptional modulation and genome-wide genetic screening. These approaches can now be exploited to gain understanding of the mechanisms behind coding and non-coding diabetes-associated genetic variants, allowing more precise evaluation of their contribution to diabetes pathogenesis. Despite all the progress, genome editing in primary pancreatic islets remains difficult to achieve, an important limitation requiring further technological development.
\end{abstract}

Keywords Beta cells $\cdot$ Cell models $\cdot$ CRISPR-Cas9 $\cdot$ Diabetes $\cdot$ Genome editing $\cdot$ Human islets $\cdot$ Pancreas $\cdot$ Review $\cdot$ Stem cells

\author{
Abbreviations \\ AAV Adenovirus and adeno-associated viruses \\ Cas9 CRISPR-associated protein 9 \\ CRISPR Clustered Regularly Interspaced Short \\ Palindromic Repeats \\ gRNA Guide RNA
}

$\begin{array}{ll}\text { GWAS } & \text { Genome-wide association studies } \\ \text { HDR } & \text { Homology-directed repair } \\ \text { hESC } & \text { Human embryonic stem cell } \\ \text { hiPSC } & \text { Human induced pluripotent stem cell } \\ \text { hPSC } & \text { Human pluripotent stem cell } \\ \text { lncRNA } & \text { Long non-coding RNA }\end{array}$

GWAS Genome-wide association studies

hESC

hiPSC Human induced pluripotent stem cell

lncRNA Long non-coding RNA
Electronic supplementary material The online version of this article (https://doi.org/10.1007/s00125-019-4908-z) contains a slide of the figure for download, which is available to authorised users.

Timo Otonkoski

timo.otonkoski@helsinki.fi

1 Stem Cells and Metabolism Research Program, Faculty of Medicine, University of Helsinki, PO Box 63, (Haartmaninkatu 8), FI-00014 Helsinki, Finland

2 Present address: Centre for Genomic Regulation (CRG), The Barcelona Institute of Science and Technology, Barcelona, Spain
3 Genomics, Diabetes and Endocrinology, Lund University Diabetes Centre, CRC, Malmö, Sweden

4 Institute of Molecular Medicine, Centre of Excellence in Complex Disease Genetics, University of Helsinki, Helsinki, Finland

5 Children's Hospital, Helsinki University Hospital and University of Helsinki, Helsinki, Finland 


\section{Introduction}

Failing beta cell function is a major culprit in all forms of diabetes. In type 1 diabetes this results from an interaction between genetic predisposition and environmental factors, culminating in an immune-mediated loss of beta cells. In monogenic diabetes, insulin secretion is deficient entirely based on mutations in genes that are important for beta cell function. Type 2 diabetes is considered to result from a collision between genetic predisposition and an affluent environment, which means that type 2 diabetes develops when people no longer can increase their insulin secretion to meet the increased demands imposed by obesity and insulin resistance. Not surprisingly, most of the 403 genetic variants identified in genome-wide association studies (GWAS) to be associated with type 2 diabetes [1] have been shown to influence beta cell function. A problem with these studies is that they have considered type 2 diabetes as a relatively homogenous disease. We have recently shown that this is not the case. By measuring a few pathogenically relevant variables, we could break down type 2 diabetes into five subgroups with quite different characteristics and disease progression [2].

Variants in several genes show strong association with type 2 diabetes risk, including those in TCF7L2, SLC3OA8 and $M T N R 1 B$ [1]. Although the genetic risk of type 1 diabetes is most strongly associated with the HLA genes, more than 50 additional genes or loci have been associated with the disease, most being expressed in the pancreatic beta cells [3].
However, it is not easy to infer causality from a common genetic variant associated with either type 1 or type 2 diabetes. Therefore, functional studies using genetically defined cells in appropriate models are required. Possibilities for studying human beta cell function in vivo are limited. In order to understand the pathogenic role of diabetes-associated genetic variants, experimental beta cell models are needed. Rodent models, particularly transgenic mice, have provided a lot of valuable information but they have limitations due to obvious genetic and physiological species differences. Essentially, there are three possible ways to study human beta cells directly: (1) primary islets isolated from the pancreas of organ donors; (2) clonal human beta cell lines and (3) islet-like cells differentiated from human pluripotent stem cells (hPSCs), comprising either human embryonic stem cells (hESCs) or human induced pluripotent stem cells (hiPSCs) (see Text box).

\section{Primary human islets}

Human pancreatic islets obtained from organ donor pancreases or from pancreatic surgery are very informative, since they are obtained while the blood flow is still intact, thereby retaining functionality of the cells. Comprehensive transcriptomic profiling of such islets, together with GWAS, has facilitated extensive analysis of expression [4] and effects of genetic variation on gene expression (i.e. expression quantitative traits [eQTLs], splicing [splice QTLS], allelic

\begin{tabular}{|c|c|c|}
\hline \multicolumn{3}{|c|}{ Sources of human pancreatic beta cells for diabetes research } \\
\hline Source & Benefits & Limitations \\
\hline \multirow[t]{4}{*}{ Primary islets } & The gold standard & Poor availability \\
\hline & & High variability \\
\hline & & Difficult to maintain \\
\hline & & $\begin{array}{l}\text { Limited possibilities for genetic } \\
\text { manipulation }\end{array}$ \\
\hline \multirow{3}{*}{$\begin{array}{l}\text { Human beta cell } \\
\text { lines }\end{array}$} & Expandability & Transformed aneuploid cells \\
\hline & Glucose-stimulated insulin secretion & Limited possibilities for genome editing \\
\hline & $\begin{array}{l}\text { Transcriptomically close to primary beta } \\
\text { cells }\end{array}$ & \\
\hline \multirow{5}{*}{$\begin{array}{l}\text { Pluripotent stem } \\
\text { cell-derived islets }\end{array}$} & Expandability & Immaturity \\
\hline & Can be produced from patients' cells & Variability between stem cell lines \\
\hline & Can be used to study islet development & Limited functional life span in vitro \\
\hline & Efficient genome editing & \\
\hline & $\begin{array}{l}\text { Can be transplanted to generate } \\
\text { humanised mice }\end{array}$ & \\
\hline
\end{tabular}


imbalance [5], cis-regulatory networks [6, 7] and non-coding RNAs [8]) using collections of isolated human islets. This has enabled the discovery of numerous genes with a potential role in glucose metabolism and insulin secretion. In order to make these resources more accessible to the scientific community, the Islet Gene View was created, providing comprehensive information on gene expression in relation to diabetes status, insulin secretion, expression of other pancreatic genes and related phenotypes of interest [9]. Overlaying expression data with data on regions of open chromatin (DNase I hypersensitive sites sequencing [DNase-seq], assay for transposaseaccessible chromatin sequencing [ATAC-seq]) [10], histone modifications (chromatin immunoprecipitation sequencing [ChIP-seq]) [11] and spatial chromatin organisation data (HiC, Capture-C or 4-C methods) [12] can facilitate a better understanding of the genomic regulation critical for appropriate islet function.

Pancreatic islets consist of multiple cell types, each with distinctive functions. Performing single-cell mRNA sequencing on different cell types, including alpha, beta, gamma, delta and epsilon cells from adult and fetal pancreases, can facilitate the identification of unique cell-specific expression profiles [13-15] in the hope of distinguishing profiles between type 2 diabetes and non-diabetic donors [16, 17]. Interestingly, key type 2 diabetes genes reported from previous studies, such as TCF7L2 and others, were missing in these data, suggesting that these studies may have been underpowered or that some of the earlier studies using bulk RNA sequencing may have been confounded by signals from cells other than endocrine cells. In addition, these differences are likely to reflect the technical limitations of single-cell mRNA sequencing technologies: limited number of cells analysed and a low gene detection rate.

Different viral vectors have been exploited to perform overexpression and perturbation experiments in human islets. Lentiviruses, adenovirus and adeno-associated viruses (AAVs) carrying cDNA-expressing constructs or short hairpin RNA (shRNA) have been transduced to human islet cells [7]. However, genome editing using site-directed endonucleases in primary islets has not previously been reported, possibly because this approach may be challenging due to a variety of factors, including poor delivery efficiency to intact islets, the quiescent nature of the cells or the sensitivity of the cells to these manipulations. These limitations might be overcome in the future with use of optimised Clustered Regularly Interspaced Short Palindromic Repeats (CRISPR)CRISPRassociated protein 9 (Cas9) approaches, such as those tailored for primary cells (e.g. Guide Swap [18]), the use of Cas9 base editors [19] or improved delivery methods to intact islets (e.g. smaller Cas9 delivered using AAVs). An alternative possibility would be the use of bioengineered human pseudoislets [20], in which dissociated cells are treated with CRISPRCas9 and then reaggregated.

\section{Human beta cell lines}

Human beta cell lines have been a long-sought resource for diabetes research. Finally, Scharfmann and co-workers succeeded in generating stable human beta cell lines from human fetal pancreatic cells using the SV40LT oncogene under the insulin promoter [21]. The first line, EndoC- $\beta \mathrm{H} 1$, has now been adopted for use in many laboratories and generally accepted as a stable glucose-responsive human beta cell line, which has numerous applications, ranging from studies of insulin secretion to studies of beta cell damage [22]. The line has obvious advantages, such as the possibility to expand it in an unlimited manner and its responsiveness to glucose at a physiological range. Additional EndoC- $\beta \mathrm{H}$ lines 2 and 3 have been developed in which the oncogene can be removed, resulting in cell-cycle arrest and increased insulin secretion in response to glucose. EndoC- $\beta \mathrm{H}$ cells are amenable to different perturbation experiments since they can be transfected chemically and electroporated with plasmid vectors or small interfering RNA (siRNA) molecules, or transduced with viral vectors [22]. However, it is challenging to genetically modify this cell line at a clonal level, given its slow growth rate and low clonal efficiency. Furthermore, it should be remembered that these are transformed aneuploid cells that cannot be taken as a direct counterpart of the primary beta cell.

\section{hPSC-based models}

The third option for achieving human beta cells for experimental studies is based on the differentiation of hPSCs. The first report describing successful differentiation of hESCs to pancreatic endocrine cells was published in 2006 by D'Amour et al [23]. Since then, the stepwise protocols that are needed to mimic normal pancreatic differentiation have been further optimised, resulting in methods that lead to the generation of large numbers of islet-like cell aggregates consisting predominantly of beta cells that are capable of responding to physiological insulin secretagogues [24, 25]. Metabolic maturation of the cells, measured as robust glucose-stimulated insulin secretion, is still difficult to achieve in vitro, but the immature cells do have a remarkable capacity for maturation after implantation into rodents. This enables the generation of 'humanised' mouse models, where the implanted human beta cells are responsible for glycaemic control in the mouse. We have recently reviewed the possibilities of stem cell strategies for the modelling of beta cell pathophysiology elsewhere [26]. Organoid technologies have evolved rapidly, enabling the generation of self-renewing 'mini-organs' from both primary tissue stem cells and pluripotent stem cells [27]. Pancreatic organoids have also been described, although this technique remains unproven as a practical solution for the efficient expansion and differentiation of pancreatic progenitors. 
The use of stem cells to generate human beta cells is an optimal approach for several reasons (see Text box); a key advantage is the possibility of using patient-derived iPSCs as starting material, making it possible to recapitulate functional features that are specific for an individual's genotype. The recapitulation of normal organ development also allows us to model developmental defects, which is not possible if end-stage differentiated islets of beta cells are used. However, because of the high variability between individual iPSC lines, findings need to be replicated in a large number of lines derived from different donors and an equally large number of control individuals [28]. Considering the demanding differentiation procedures, this is a formidable challenge. Therefore, genome editing of stem cells is an attractive possibility for reducing the variability between cell lines and focusing on the impact of specific genetic variants (Fig. 1). Different genome engineering technologies, like zinc finger nucleases (ZFN) [29] and transcription activator like effector nucleases (TALEN) [30], have been used successfully on hPSCs but these have been superseded by CRISPR-Cas9

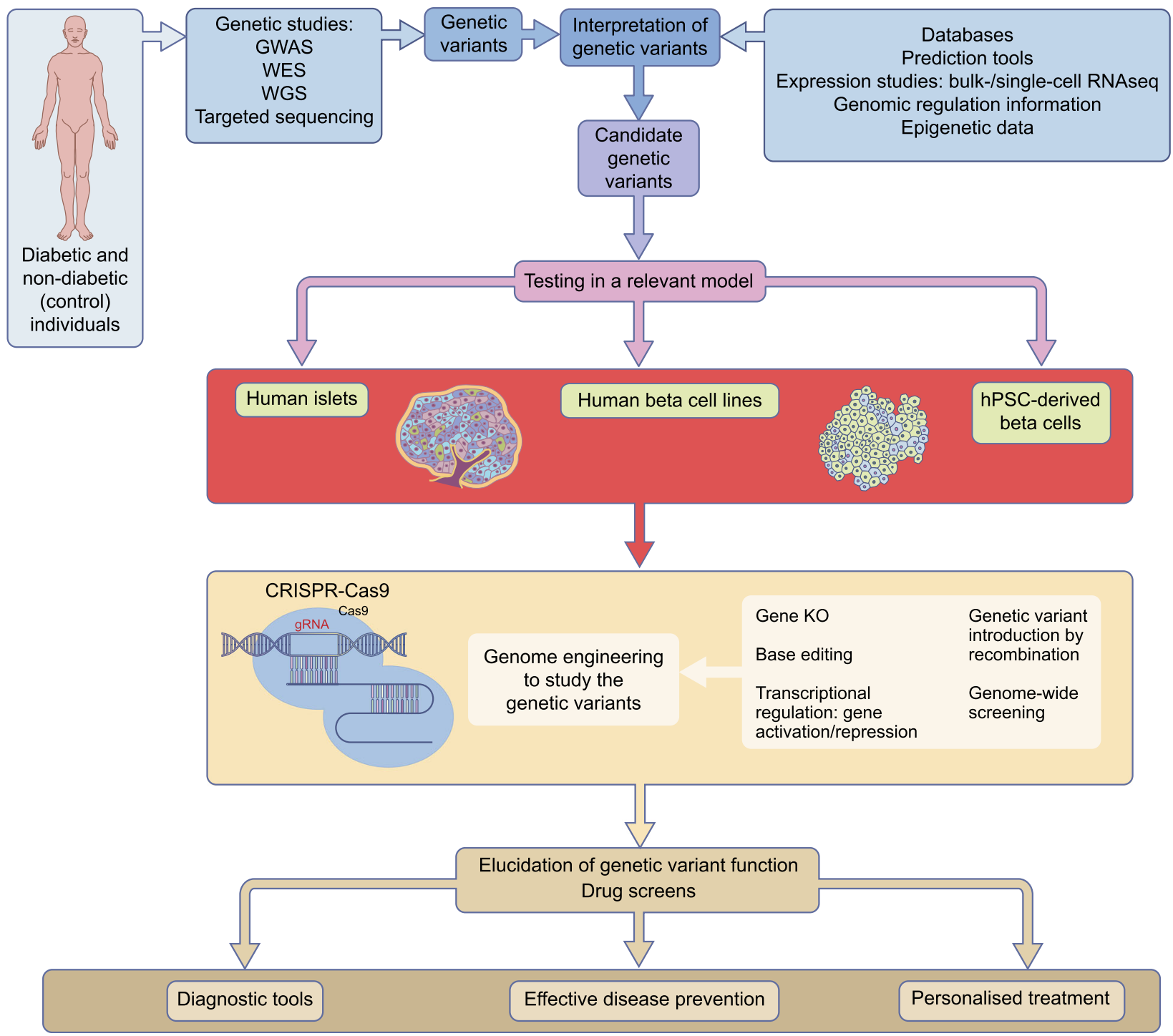

Fig. 1 The central role of human beta cell models in the functional analysis of diabetes-associated genotypes. Genetic variants identified in people affected with diabetes (via genetic studies) can be interpreted using integrated functional genomic data (databases, prediction tools, epigenetic data, etc.). Candidate genetic variants require validation in relevant experimental models. Genome engineering technologies (e.g. gene knockout, base editing and genetic recombination) facilitate the genetic manipulation of cellular models to elucidate the role of the candidate genetic variants. In particular, genome engineering using CRISPR-Cas9 systems (consisting of two parts: a Cas9 endonuclease protein and
gRNAs) have recently opened exciting new avenues for interrogating the functional impact of diabetes-associated genetic variants. These genome engineered models might also be utilised as scalable drug-screening platforms. Understanding the functional impact of diabetes-associated genetic variants will allow better diagnosis and stratification of diabetes cases, implementation of more effective interventions for diabetes prevention and more optimal personalised treatment for people affected by diabetes. KO, knockout; RNAseq, RNA sequencing; WES, whole exome sequencing; WGS, whole genome sequencing. This figure is available as a downloadable slide 
technology, which has dramatically improved the possibilities of genome editing.

\section{Genome editing with CRISPR-Cas9}

Genome engineering is based on the use of sequence-specific endonucleases that introduce DNA double-strand breaks in a targeted genomic sequence. These targeted cuts may disrupt that particular sequence, possibly resulting in a gene knockout, or stimulate homologous recombination with exogenous DNA templates (homology-directed repair [HDR]), which can be exploited to create knockins in order to correct or introduce point mutations.

The genome editing revolution started in 2013, when the first CRISPR-Cas9 system was engineered to work in mammalian cells [31-33]. The system consists of two parts: a Cas9 endonuclease protein and short RNA molecules, called guide RNAs (gRNAs). The latter are loaded into the Cas9 protein to form a ribonucleoprotein complex that seeks and cleaves DNA sequences complementary to the gRNA sequence. CRISPR-Cas9 systems have been widely used to disrupt genes in different cell lines and organisms. CRISPR technology and its multiple applications have been thoroughly reviewed elsewhere [34, 35].

The overall gene disruption efficiency depends on many factors, including the expression level of Cas9 protein, the sequence and quality features of the gRNA and the cellcycle phase. To generate a reliable gene knockout, gRNAs should be designed to target all the splice variants of the gene of interest, including essential functional domains and considering possible alternative translation start sites. Targeting regions directly downstream of the start codon is also used but this may result in hypomorphic alleles due to alternative start sites. Defined genomic regions can also be excised by using pairs of gRNAs to generate a large deletion. This is particularly useful for studying the importance of non-coding and regulatory elements $[12,36]$. The generation of large deletions in the genome might have unintended consequences if, for example, non-annotated regulatory regions are also removed. For this reason, CRISPR-based genome manipulation experiments should be carefully planned to include appropriate controls and alternative modification approaches (e.g. point mutation introduction, base editing).

The introduction or correction of particular point mutations using CRISPR-Cas9 is an important application by which to investigate the role of particular genetic variants. To achieve this, a Cas9-mediated cut is generated adjacent to the position of interest while providing a homologous donor template with the intended nucleotide change, usually in the form of a short single-stranded DNA oligo, that will recombine by HDR [37]. Another approach is the use of engineered Cas9 versions that work as base editors, which convert DNA bases (transitions C to $\mathrm{T}$ and $\mathrm{A}$ to $\mathrm{G}$ ) without cleaving the DNA, thereby avoiding the risks of undesired on- and off-target cut effects [19]. An interesting advantage of base editing is its high efficiency in quiescent cells [38], in contrast to the inefficiency of HDR in non-dividing cells. This could be exploited to manipulate adult human beta cells, which are largely quiescent.

Exciting novel experimental possibilities have been enabled by the engineering of catalytically inactive ('dead') Cas9 proteins with transcriptional activator and repressor domains (e.g. repeats of VP16, Krüppel associated box [KRAB], or epigenetic modulators such as DNA methyltransferase $3 \alpha$ [DNMT3A], p300, etc.) $[39,40]$. These Cas9-based effectors make it possible to perform hitherto unfeasible targeted transcriptional modulation and epigenome modification of endogenous loci. One conceptual advance has been the development of unbiased CRISPR-Cas9based whole-genome genetic screenings, enabling genomewide-scale gene knockouts, deletion of regulatory regions and transcriptional activation or repression [41].

CRISPR-Cas9 tools offer unprecedented experimental approaches to dissect the mechanisms of beta cell function in health and disease. They can be used to modulate transcription and manipulate the genome in human beta cell lines $[12,36]$. They also enable the generation of novel genetically modified animal models (not only restricted to rodents), allowing comparison and the conservation of beta cell function mechanism across species. CRISPR-Cas9 approaches are also used on hPSCs to generate gene knockouts [42], correct and introduce point mutations [43], engineer fluorescent reporter cell lines and modulate transcription.

\section{Proof of principle}

Modelling of monogenic diabetes Monogenic diabetes presents at a young age due to mutations in a single gene, leading to impaired function of the pancreatic beta cells. The exact molecular mechanisms leading to beta cell failure can be addressed in carefully planned cellular models. A particular genetic modification (e.g. knockout, knockin) can be generated in a well-differentiating, healthy hPSC line or a candidate mutation can be corrected in patient-derived hiPSCs. Resulting isogenic cell line pairs have the same genetic background and similar differentiation properties, while being discordant only for the mutation of interest.

Genome editing has been used on hPSCs to knock out genes critical for pancreatic and beta cell development (e.g. PDX1, NEUROG3, ARX, GLIS3, NEUROD1), thus reproducing with human cells previous findings made in transgenic mouse studies [42, 44, 45]. Furthermore, correction of point mutations in patient-derived hiPSCs has been exploited to interrogate the disease mechanism in rare cases of neonatal diabetes, such as those caused by mutations in STAT3 and 
GATA6 genes [43, 45]. Recently, this strategy was used to show how diabetogenic mutations in the INS gene lead to chronic endothelial reticulum stress-associated failure of beta cell growth [46].

Modelling of polygenic diabetes More than 400 SNPs associated with type 2 diabetes and related traits have been identified thus far. The functional elucidation of these loci has, however, been largely elusive [1]. For the last 100 years diabetes has been diagnosed by measuring one metabolite, glucose. This has of course identified individuals with elevated glucose levels but provided little information on underlying pathogenic causes. By including six variables (age at diagnosis, BMI, $\mathrm{HbA}_{1 \mathrm{c}}$, GAD autoantibodies, C-peptide and glucose [for estimation of insulin secretion, HOMA-B and insulin-sensitivity, HOMA-IS]) in a clustering analysis of individuals with newly diagnosed diabetes, we could break down classical type 2 diabetes into five distinct subgroups, with better prediction of disease progression and outcome [2]. These clusters also seem to differ in genetic background.

Therefore, gene silencing and functional characterisation or genome editing of type 2 diabetes risk alleles or deleting regulatory regions surrounding these variants in human beta cell models, followed by RNA sequencing (RNAseq) and/ or implementation of spatial chromatin organisation methods (4C/Hi-C/Capture-C), could facilitate a better understanding of the functional effects of these variants. In the human beta cell line EndoC- $\beta \mathrm{H} 1$, the silencing of candidate genes selected from 75 type 2 diabetes-associated loci revealed 45 genes involved in beta cell function including ARL15, ZMIZ1, and THADA [47]. Beta cell-specific long non-coding RNA (lncRNA) transcript knockdown and coexpression analysis demonstrated the role of lncRNAs that collaborate with transcription factors to regulate beta cell-specific transcriptional networks. Further, PLUTO (also known as $P L U T$ ), the antisense transcript of the $P D X 1$ gene, modulates the chromatin structure and transcription of $P D X 1$. Both PDX1 and PLUTO are downregulated in islets from hyperglycaemic donors [36]. One of the strongest association signals for type 2 diabetes is the rs7903146 T allele SNP in the TCF7L2 gene, and researchers have tried hard to understand the molecular mechanism behind this association. Some previous studies have reported increased chromatin accessibility and episomal enhancer activity for the T allele SNP and higher TCF7L2 expression was found in carriers of the TT genotype with type 2 diabetes [48, 49]. Recently, it was shown that CRISPR-mediated deletion of the region harbouring the type 2 diabetes risk SNP rs7903146 leads to a decrease in TCF7L2 mRNA levels, while targeting it with a CRISPR transcriptional activator had the opposite effect. These findings further indicate that this region constitutes an enhancer regulating TCF $7 L 2$ expression in human islet cells [12].
The interrogation of type 2 diabetes risk variants could be performed in an unbiased manner by combining CRISPRCas9-based genome-wide genome and epigenome editing with single-cell omics to assess the transcriptional and functional outcome of the variants [41]. Genes associated with type 2 diabetes risk have been knocked out in hPSCs to elucidate their putative role and mechanism predisposing to the disease (e.g. CDKAL1, KCNQ1) [50]. Further improvements on the functionality of stem cell-derived beta-like cells will provide better chances to unravel the functional impact of type 2 diabetes risk variants on beta cell development and physiology. First examples of genome-edited hPSC models to elucidate the role of specific type 2 diabetes-associated SNPs are starting to appear, as exemplified by a report where CRISPRCas9-edited hiPSCs and EndoC- $\beta \mathrm{H} 1$ cells were used to investigate the mechanisms of a protective zinc transporter 8 (Znt8, SLC30A8) variant [51].

Outlook It is easy to predict that the recent technological developments in human cellular models combined with targeted genome modification will lead to a boom in functional genomic studies of diabetes during the coming years. The task is formidable because of the many disease-associated loci in non-coding DNA regions. Ingenious use of CRISPR-Cas9 and similar techniques will undoubtedly speed up the understanding of interplay between type 1 diabetes and type 2 diabetes risk-associated genetic variants and their functional role in predisposing to the disease. These approaches will also be used in drug screens, enhancing the development of targeted means for personalised treatment.

Acknowledgements Open access funding provided by University of Helsinki including Helsinki University Central Hospital.

Funding The authors' work in this area has been supported by grants from the Academy of Finland, The Novo Nordisk Foundation and the Sigrid Jusélius Foundation. TO is also funded by the Innovative Medicines Initiative 2 Joint Undertaking under grant agreement no. 115797 (INNODIA), which receives support from the European Union's Horizon 2020 research and innovation programme and the European Federation of Pharmaceutical Industries and Associations (EFPIA), JDRF and the Leona M. and Harry B. Helmsley Charitable Trust.

Duality of interest The authors declare that there is no duality of interest associated with this manuscript.

Contribution statement All authors were responsible for drafting and revising this review article. All authors approved the final version to be published.

Open Access This article is distributed under the terms of the Creative Commons Attribution 4.0 International License (http:// creativecommons.org/licenses/by/4.0/), which permits unrestricted use, distribution, and reproduction in any medium, provided you give 
appropriate credit to the original author(s) and the source, provide a link to the Creative Commons license, and indicate if changes were made.

\section{References}

1. Mahajan A, Taliun D, Thurner M et al (2018) Fine-mapping type 2 diabetes loci to single-variant resolution using high-density imputation and islet-specific epigenome maps. Nat Genet 50(11):15051513. https://doi.org/10.1038/s41588-018-0241-6

2. Ahlqvist E, Storm P, Käräjämäki A et al (2018) Novel subgroups of adult-onset diabetes and their association with outcomes: a datadriven cluster analysis of six variables. Lancet Diabetes Endocrinol 8587(18):1-9. https://doi.org/10.1016/S2213-8587(18) 30051-2

3. Eizirik DL, Sammeth M, Bouckenooghe T et al (2012) The human pancreatic islet transcriptome: expression of candidate genes for type 1 diabetes and the impact of pro-inflammatory cytokines. PLoS Genet 8(3):e1002552. https://doi.org/10.1371/journal.pgen. 1002552

4. Taneera J, Fadista J, Ahlqvist E et al (2015) Identification of novel genes for glucose metabolism based upon expression pattern in human islets and effect on insulin secretion and glycemia. Hum Mol Genet 24(7):1945-1955. https://doi.org/10.1093/hmg/ddu610

5. Fadista J, Vikman P, Laakso EO et al (2014) Global genomic and transcriptomic analysis of human pancreatic islets reveals novel genes influencing glucose metabolism. Proc Natl Acad Sci 111(38):13924-13929. https://doi.org/10.1073/pnas.1402665111

6. Pasquali L, Gaulton KJ, Rodríguez-Seguí SA et al (2014) Pancreatic islet enhancer clusters enriched in type 2 diabetes riskassociated variants. Nat Genet 46(2):136-143. https://doi.org/10. 1038/ng.2870

7. van de Bunt M, Manning Fox JE, Dai X et al (2015) Transcript expression data from human islets links regulatory signals from genome-wide association studies for type 2 diabetes and glycemic traits to their downstream effectors. PLoS Genet 11(12):e1005694. https://doi.org/10.1371/journal.pgen.1005694

8. Morán I, Akerman I, van de Bunt M et al (2012) Human $\beta$ cell transcriptome analysis uncovers lncRNAs that are tissue-specific, dynamically regulated, and abnormally expressed in type 2 diabetes. Cell Metab 16(4):435-448. https://doi.org/10.1016/j.cmet. 2012.08.010

9. Asplund O, Storm P, Ottosson-Laakso E et al (2018) Islet Gene View - a tool to facilitate islet research. bioRxiv 435743. https:// doi.org/10.1101/435743

10. Varshney A, Scott LJ, Welch RP et al (2017) Genetic regulatory signatures underlying islet gene expression and type 2 diabetes. Proc Natl Acad Sci 114(9):2301-2306. https://doi.org/10.1073/ pnas. 1621192114

11. Roman TS, Cannon ME, Vadlamudi S et al (2017) A type 2 diabetes-associated functional regulatory variant in a pancreatic islet enhancer at the ADCY5 locus. Diabetes 66(9):2521-2530. https://doi.org/10.2337/db17-0464

12. Miguel-Escalada I, Bonàs-Guarch $\mathrm{S}$, Cebola I et al (2018) Human pancreatic islet 3D chromatin architecture provides insights into the genetics of type 2 diabetes. bioRxiv 400291. https://doi.org/10. $1101 / 400291$

13. Blodgett DM, Nowosielska A, Afik S et al (2015) Novel observations from next-generation rna sequencing of highly purified human adult and fetal islet cell subsets. Diabetes 64(9):3172-3181. https:// doi.org/10.2337/db15-0039

14. Wang YJ, Schug J, Won K-J et al (2016) Single-cell transcriptomics of the human endocrine pancreas. Diabetes 65(10):3028-3038. https://doi.org/10.2337/db16-0405
15. Dominguez Gutierrez G, Kim J, Lee A-H et al (2018) Gene signature of the human pancreatic $\varepsilon$-cell. Endocrinology 159(12):40234032. https://doi.org/10.1210/en.2018-00833

16. Segerstolpe Å, Palasantza A, Eliasson P et al (2016) Single-cell transcriptome profiling of human pancreatic islets in health and type 2 diabetes. Cell Metab 24(4):593-607. https://doi.org/10.1016/j. cmet.2016.08.020

17. Xin Y, Kim J, Okamoto $\mathrm{H}$ et al (2016) RNA sequencing of single human islet cells reveals type 2 diabetes genes. Cell Metab 24(4): 608-615. https://doi.org/10.1016/J.CMET.2016.08.018

18. Ting PY, Parker AE, Lee JS et al (2018) Guide Swap enables genome-scale pooled CRISPR-Cas9 screening in human primary cells. Nat Methods 15(11):941-946. https://doi.org/10.1038/ s41592-018-0149-1

19. Rees HA, Liu DR (2018) Base editing: precision chemistry on the genome and transcriptome of living cells. Nat Rev Genet 19(12): 770-788. https://doi.org/10.1038/s41576-018-0059-1

20. Yu Y, Gamble A, Pawlick R et al (2018) Bioengineered human pseudoislets form efficiently from donated tissue, compare favourably with native islets in vitro and restore normoglycaemia in mice. Diabetologia 61(9):2016-2029. https://doi.org/10.1007/ s00125-018-4672-5

21. Ravassard P, Hazhouz Y, Pechberty S et al (2011) A genetically engineered human pancreatic $\beta$ cell line exhibiting glucoseinducible insulin secretion. J Clin Invest 121(9):3589-3597. https://doi.org/10.1172/JCI58447

22. Tsonkova VG, Sand FW, Wolf XA et al (2018) The EndoC- $\beta \mathrm{H} 1$ cell line is a valid model of human beta cells and applicable for screenings to identify novel drug target candidates. Mol Metab 8: 144-157. https://doi.org/10.1016/j.molmet.2017.12.007

23. D'Amour KAKA, Bang AGAG, Eliazer S et al (2006) Production of pancreatic hormone-expressing endocrine cells from human embryonic stem cells. Nat Biotechnol 24(11):1392-1401. https://doi. org $/ 10.1038 /$ nbt1259

24. Rezania A, Bruin JE, Arora P et al (2014) Reversal of diabetes with insulin-producing cells derived in vitro from human pluripotent stem cells. Nat Biotechnol 32(11):1121-1133. https://doi.org/10. 1038/nbt.3033

25. Velazco-Cruz L, Song J, Maxwell KG et al (2019) Acquisition of dynamic function in human stem cell-derived $\beta$ cells. Stem Cell Reports 12(2):351-365. https://doi.org/10.1016/j.stemcr.2018.12. 012

26. Balboa D, Saarimäki-Vire J, Otonkoski T (2019) Human pluripotent stem cells for the modelling of pancreatic $\beta$-cell pathology. Stem Cells 62(1):87-98. https://doi.org/10.1002/stem.2913

27. McCauley HA, Wells JM (2017) Pluripotent stem cell-derived organoids: using principles of developmental biology to grow human tissues in a dish. Development 144(6):958-962. https://doi. org/10.1242/dev.140731

28. Kyttälä A, Moraghebi R, Valensisi C et al (2016) Genetic variability overrides the impact of parental cell type and determines iPSC differentiation potential. Stem Cell Rep 6(2):200-212. https://doi. org/10.1016/j.stemcr.2015.12.009

29. Lombardo A, Genovese P, Beausejour CM et al (2007) Gene editing in human stem cells using zinc finger nucleases and integrase-defective lentiviral vector delivery. Nat Biotechnol 25(11):1298-1306. https://doi.org/10.1038/nbt1353

30. Ding Q, Lee Y-KK, Schaefer EA et al (2012) A TALEN genomeediting system for generating human stem cell-based disease models. Cell Stem Cell 12(2):1-14. https://doi.org/10.1016/j.stem. 2012.11.011

31. Cong L, Ran FA, Cox D et al (2013) Multiplex genome engineering using CRISPR/Cas systems. Science 339(6121):819-823. https:// doi.org/10.1126/science. 1231143 
32. Mali P, Yang L, Esvelt KM et al (2013) RNA-guided human genome engineering via Cas9. Science 339(6121):823-826. https:// doi.org/10.1126/science.1232033

33. Jinek M, East A, Cheng A, Lin S, Ma E, Doudna J (2013) RNAprogrammed genome editing in human cells. eLIFE 2:e0471. https://doi.org/10.7554/eLife.00471

34. Hsu PD, Lander ES, Zhang F (2014) Development and applications of CRISPR-Cas9 for genome engineering. Cell 157(6):1262-1278. https://doi.org/10.1016/j.cell.2014.05.010

35. Komor AC, Badran AH, Liu DR (2017) CRISPR-based technologies for the manipulation of eukaryotic genomes. Cell 168(1-2): 20-36. https://doi.org/10.1016/j.cell.2016.10.044

36. Akerman I, Tu Z, Beucher A et al (2017) Human pancreatic $\beta$ cell lncRNAs control cell-specific regulatory networks. Cell Metab 25(2):400-411. https://doi.org/10.1016/j.cmet.2016.11.016

37. Paquet D, Kwart D, Chen A et al (2016) Efficient introduction of specific homozygous and heterozygous mutations using CRISPR/ Cas9. Nature 533(7601):1-18. https://doi.org/10.1038/nature17664

38. Yeh W-H, Chiang H, Rees HA, Edge ASB, Liu DR (2018) In vivo base editing of post-mitotic sensory cells. Nat Commun 9(1):2184. https://doi.org/10.1038/s41467-018-04580-3

39. Hilton IB, D'Ippolito AM, Vockley CM et al (2015) Epigenome editing by a CRISPR-Cas9-based acetyltransferase activates genes from promoters and enhancers. Nat Biotechnol 33(5):510-517. https://doi.org/10.1038/nbt.3199

40. Balboa D, Weltner J, Eurola S, Trokovic R, Wartiovaara K, Otonkoski T (2015) Conditionally stabilized dCas9 activator for controlling gene expression in human cell reprogramming and differentiation. Stem Cell Rep 5(3):448-459. https://doi.org/10.1016/ j.stemcr.2015.08.001

41. Doench JG (2017) Am I ready for CRISPR? A user's guide to genetic screens. Nat Rev Genet 19(2):67-80. https://doi.org/10. 1038/nrg.2017.97

42. Zhu Z, Li QV, Lee K et al (2016) Genome editing of lineage determinants in human pluripotent stem cells reveals mechanisms of pancreatic development and diabetes. Cell Stem Cell 18(6):755768. https://doi.org/10.1016/j.stem.2016.03.015

43. Saarimäki-Vire J, Balboa D, Russell MA et al (2017) An activating STAT3 mutation causes neonatal diabetes through premature induction of pancreatic differentiation. Cell Rep 19(2):281-294. https:// doi.org/10.1016/j.celrep.2017.03.055
44. McGrath PS, Watson CL, Ingram C, Helmrath MA, Wells JM (2015) The basic helix-loop-helix transcription factor NEUROG3 is required for development of the human endocrine pancreas. Diabetes 64(7):2497-2505. https://doi.org/10.2337/db14-1412

45. Tiyaboonchai A, Cardenas-Diaz FL, Ying L et al (2017) GATA6 plays an important role in the induction of human definitive endoderm, development of the pancreas, and functionality of pancreatic $\beta$ cells. Stem Cell Reports 8(3):589-604. https://doi.org/10.1016/j. stemcr.2016.12.026

46. Balboa D, Saarimäki-Vire J, Borshagovski D et al (2018) Insulin mutations impair $\beta$-cell development in a patient-derived iPSC model of neonatal diabetes. eLIFE 7:e38519. https://doi.org/10. 7554/eLife.38519

47. Thomsen SK, Ceroni A, van de Bunt M et al (2016) Systematic functional characterization of candidate causal genes for type 2 diabetes risk variants. Diabetes 65(12):3805-3811. https://doi.org/ $10.2337 / \mathrm{db} 16-0361$

48. Gaulton KJ, Nammo T, Pasquali L et al (2010) A map of open chromatin in human pancreatic islets. Nat Genet 42(3):255-259. https://doi.org/10.1038/ng.530

49. Lyssenko V, Lupi R, Marchetti P et al (2007) Mechanisms by which common variants in the TCF7L2 gene increase risk of type 2 diabetes. J Clin Invest 117(8):2155-2163. https://doi.org/10.1172/ JCI30706

50. Zeng H, Guo M, Zhou T et al (2016) An isogenic human ESC platform for functional evaluation of genome-wide-associationstudy-identified diabetes genes and drug discovery. Cell Stem Cell 19(3):326-340. https://doi.org/10.1016/j.stem.2016.07.002

51. Dwivedi OP, Lehtovirta M, Hastoy B et al (2018) Loss of ZnT8 function protects against diabetes by enhanced insulin secretion. bioRxiv 436030. https://doi.org/10.1101/436030

Publisher's note Springer Nature remains neutral with regard to jurisdictional claims in published maps and institutional affiliations. 